\title{
The prevalence of potentially inappropriate medications for patients with heart failure (PIMHF) use and its association with non-cardiovascular co-morbidities in Thai patients with heart failure
}

\author{
Kittipak Jenghua ${ }^{1}$ \\ ${ }^{1}$ University of Phayao School of Pharmaceutical Sciences
}

October 1, 2020

\begin{abstract}
Background: Several medications may worsen heart failure (HF), and they are considered as potentially inappropriate medications for patients with heart failure (PIMHF). No studies have reported the prevalence of PIMHF use and its associated factors in Thai HF patients. Objective: To determine the prevalence of PIMHF use and identify the factors associated with PIMHF use. Materials and Methods: A cross-sectional analytical study was conducted using data on HF patients obtained from the electronic medical databases (EMD) of two hospitals, including a secondary- and a tertiary-care hospital. Data collected included demographics, diagnoses, and medication items prescribed during 2016-2019. The prevalence of PIMHF use identified by the Thailand list of PIMHF was determined. Patient and clinical factors were examined for association with PIMHF use by calculating the adjusted odds ratio (aOR) and $95 \%$ confidence interval (95\% CI) using a binary logistic regression analysis. Results: From the EMD, 972 and 2,888 eligible HF patients from a secondary- and a tertiary-care hospital, respectively, were included in this study. The prevalence of PIMHF use was $45.16 \%$ and $33.07 \%$ at a secondary- and a tertiary-care hospital, respectively. The PIMHF distribution appeared similar between the two study hospitals, with oral corticosteroids being the most frequently prescribed, followed by NSAIDs, COX-2 inhibitors, and thiazolidinediones. The factors associated with PIMHF use were non-cardiovascular (non-CVD) co-morbidities, including diabetes mellitus (aOR = 1.68, 95\%CI = 1.42-1.99), chronic pulmonary diseases $(\mathrm{aOR}=2.69,95 \% \mathrm{CI}=2.07-3.48)$, connective tissue diseases $(\mathrm{aOR}=7.16,95 \% \mathrm{CI}=3.09-16.57)$, and cancer $(\mathrm{aOR}=1.97,95 \% \mathrm{CI}=1.20-3.22)$. Conclusion: PIMHF use was prevalent in Thai HF patients and associated with certain non-CVD co-morbidities. A careful prescription and a review of medication use should focus on HF patients with specific non-CVD co-morbidities. Keywords: Heart failure; Potentially inappropriate medications; Thailand criteria; Prevalence; Factors associated
\end{abstract}

\section{Dr. Kittipak Jenghua}

Department of Pharmaceutical Care

School of Pharmaceutical Sciences, University of Phayao

Tambon Maeka, Muang District, Phayao, 56000

October 1, 2020

Dear Dr. Charles Young,

We wish to submit an original research article entitled "The prevalence of potentially inappropriate medications for patients with heart failure (PIMHF) use and its association with noncardiovascular co-morbidities in Thai patients with heart failure " for consideration by the International Journal of Clinical Practice (IJCP). 
We confirm that this work is original and has not been published elsewhere, nor is it currently under consideration for publication elsewhere.

In this paper, we report on evaluating the prevalence of potentially inappropriate medications for patients with heart failure (PIMHF) use and attempt to indicate co-morbidities associated with PIMHF use. This is significant because heart failure is common in clinical practice and selection of medication for treating co-morbidities accompanying HF has become complicated as several medications, which is considered as PIMHF, may worsen HF, leading to hospitalization. Recently, we have developed a list of PIMHF containing 47 medication items to use as a screening tool for medication use in Thai HF patients. Thus, we conducted this study to reveal the magnitude of the problem on PIMHF prescription in HF patients with co-morbidities in real clinical practice. We hope that the findings of this study can lead to develop awareness of medication use in HF patients having other co-morbidities among healthcare personnel.

We would also like to inform that our study has been reviewed and approved by the Institutional Review Board Committee on human research at Chiangkham hospital (protocol number: 008/2562) and at Lampang hospital (protocol number: 84/62). Besides, our manuscript has been professionally proofread by trusted services as an attached certificate.

We have no conflicts of interest to disclose.

Please address all correspondence concerning this manuscript to me atkittipak.je@up.ac.th

Thank you for your consideration of this manuscript.

Sincerely yours,

Kittipak Jenghua

\section{Hosted file}

Credit author statement 01102020.pdf available at https://authorea.com/users/363663/articles/ 484353-the-prevalence-of-potentially-inappropriate-medications-for-patients-with-heartfailure-pimhf-use-and-its-association-with-non-cardiovascular-co-morbidities-in-thaipatients-with-heart-failure

\section{Hosted file}

Title page information 01102020.pdf available at https://authorea.com/users/363663/articles/ 484353-the-prevalence-of-potentially-inappropriate-medications-for-patients-with-heartfailure-pimhf-use-and-its-association-with-non-cardiovascular-co-morbidities-in-thaipatients-with-heart-failure

\section{Hosted file}

Manuscript 01102020.pdf available at https://authorea.com/users/363663/articles/484353-theprevalence-of-potentially-inappropriate-medications-for-patients-with-heart-failurepimhf-use-and-its-association-with-non-cardiovascular-co-morbidities-in-thai-patientswith-heart-failure

\section{Hosted file}

Ethics 01102020.pdf available at https://authorea.com/users/363663/articles/484353-theprevalence-of-potentially-inappropriate-medications-for-patients-with-heart-failurepimhf-use-and-its-association-with-non-cardiovascular-co-morbidities-in-thai-patientswith-heart-failure 